\title{
MILLIMETER-WAVE SEARCHES FOR COLD DUST AND MOLECULAR GAS AROUND T TAURI STARS IN MBM 12
}

\author{
Michiel R. Hogerheijde, ${ }^{1,2}$ Ray Jayawardhana, ${ }^{2,3}$ Doug Johnstone, ${ }^{4}$ \\ Geoffrey A. Blake, ${ }^{5,6}$ AND JaCQueline E. Kessler ${ }^{6}$ \\ Received 2002 April 15; accepted 2002 September 4
}

\begin{abstract}
We report results of a sensitive search for cold dust and molecular gas in the disks around eight $\mathrm{T}$ Tauri stars in the high-latitude cloud MBM 12. Interferometric observations of $3 \mathrm{~mm}$ continuum emission in five fields containing six of the objects and literature values for the remaining two limit the disk masses to $M_{\text {disk }}<0.04-0.09 M_{\odot}$ (gas plus dust), for a gas-to-dust mass ratio of 100 and a distance of 275 pc. By coadding the $3 \mathrm{~mm}$ data of our five fields, we set an upper limit to the average disk mass of $\bar{M}_{\text {disk }}(N=5)<0.03$ $M_{\odot}$. Simultaneous observation of the CS $J=2-1$ and the $\mathrm{N}_{2} \mathrm{H}^{+} 1-0$ lines show no emission. Single-dish observations of the ${ }^{13} \mathrm{CO} 2-1$ line limit the disk mass to $(5-10) \times 10^{-4} M_{\odot}$ for a standard $\mathrm{CO}$ abundance of $2 \times 10^{-4}$. Depletion of CO by up to 2 orders of magnitude through freezing out or photodissociation can reconcile these limits. These mass limits lie within the range found in the Taurus-Auriga and $\rho$ Oph star-forming regions $\left(0.001-0.3 M_{\odot}\right)$ and preclude conclusions about possible decrease in disk mass over the 1-2 Myr age range spanned by the latter two regions and MBM 12. Our observations can exclude the presence in MBM 12 of T Tauri stars with relatively bright and massive disks such as T Tau, DG Tau, and GG Tau.
\end{abstract}

Key words: circumstellar matter — ISM: clouds — ISM: individual (MBM 12) — stars: formation — stars: pre-main-sequence

\section{INTRODUCTION}

Characterizing the disks of young stars is an outstanding challenge in star and planet formation research. Millimeter emission from cold dust and molecular gas has revealed rotating disks a few hundred AU in radius (Mannings \& Sargent 1997, 2000; Dutrey et al. 1996, 1998; Dutrey, Guilloteau, \& Guélin 1997; Simon, Dutrey, \& Guilloteau 2000) around T Tauri and Herbig Ae/Be stars, while millimeter and infrared observations have shown the mass in small dust particles to decrease gradually from the classical $\mathrm{T}$ Tauri to late pre-main-sequence stage (Robberto, Beckwith, \& Herbst 2000; Jayawardhana et al. 1999; Carpenter 2002). Evidence suggests that the age range of 13 Myr is pivotal in the disk's evolution, when small dust in the inner disk regions starts to clear out (Haisch, Lada, \& Lada 2001; Luhman 2001; Hartmann 2001). Some of the dust may have coagulated into larger, centimeter-sized objects, which are invisible to (sub-) millimeter or infrared observations. The fate of the gas that makes up $99 \%$ of the disk's mass is only now being investigated (Thi et al. 1999; Duvert et al. 2000; Thi et al. 2001a, 2001b; Herczeg et al. 2002; Richter et al. 2002). This paper investigates the dust and gas content of the disks around several young stars in the MBM 12 cloud.

\footnotetext{
${ }^{1}$ Steward Observatory, University of Arizona, 933 North Cherry Avenue, Tucson, AZ 85721.

2 Department of Astronomy, University of California, Berkeley, 601 Campbell Hall, Berkeley, CA 94720.

3 Department of Astronomy, University of Michigan, 830 Dennison Building, Ann Arbor, MI 48109.

${ }^{4}$ Herzberg Institute of Astrophysics, National Research Council of Canada, 5071 West Saanich Road, Victoria, BC V9E 2E7, Canada.

${ }^{5}$ Division of Geological and Planetary Sciences, M/S 150-21, California Institute of Technology, Pasadena, CA 91125.

${ }^{6}$ Division of Chemistry and Chemical Engineering, California Institute of Technology, Pasadena, CA 91125.
}

The cloud MBM 12 (distance of 275 pc; but see below) is only one of two high-latitude clouds known to harbor young stellar objects; the other being MBM 20 (Sandell, Reipurth, \& Gahm 1987). Hearty et al. (2000a) identified eight late-type young stars in MBM 12, and two mainsequence stars with unclear relation to the cloud. Luhman (2001) found four additional pre-main-sequence members from Two Micron All Sky Survey data, and estimated the cluster's age at $2_{-1}^{+3}$ Myr. Jayawardhana et al. (2001) detected mid-infrared excess toward six of the original eight objects. Based on their $\mathrm{H} \alpha$ equivalent line widths, these objects are classified as six classical T Tauri stars and two "weak-lined" T Tauri stars (Table 1 and Fig. 1). Their frequency of $K$ - (20\%) and $L$-band infrared excesses (70\%) suggests clearing of their disks (Luhman 2001). A crucial but unresolved question is how much material, if any, still resides in more extended, outer disks. Thus far, upper limits to the millimeter continuum of only two of the T Tauri stars (E02553+2018 and $\mathrm{LkH} \alpha$ 264) have been reported (Pound 1996).

The group of young stars in MBM 12 is among several nearby " "isolated" groups that have recently been identified and investigated for the nature of their disks (Jayawardhana 2000; Jayawardhana \& Greene 2001). Some of these groups (the TW Hydrae association [TWA] at $\sim 55$ pc, Kastner et al. 1997; $\eta$ Chamaeleontis cluster at $\sim 97$ pc, Mamajek, Lawson, \& Feigelson 1999) are far from obvious parent molecular clouds. This suggests ages for these stars of 5-10 $\mathrm{Myr}$, and little circumstellar disk material is expected. The association of the MBM 12 stars with cloud material supports their younger derived age, and they may represent an earlier epoch of groups like TWA and $\eta$ Cha.

Until recently, MBM 12 was thought to be the nearest star-forming cloud to the Sun, at $\sim 65 \mathrm{pc}$ (Hearty et al. 2000b). New evidence suggests it may be significantly further (Luhman 2001), at 275 pc. Idzi, Andersson, \& Uomoto (2001) found extinction at 65, 140, and 275 pc 
TABLE 1

OBSERVEd SOURCES

\begin{tabular}{|c|c|c|c|c|}
\hline Source & $\alpha(\mathrm{J} 2000.0)$ & $\delta(\mathrm{J} 2000.0)$ & $\begin{array}{c}K-L, K-N \\
\text { Excess? }\end{array}$ & Classification $^{\mathrm{b}}$ \\
\hline RXJ $0255.4+2005 \ldots \ldots$. & 025525.7 & 200453 & No & WTTS \\
\hline $\mathrm{LkH} \alpha 262 \ldots \ldots \ldots \ldots \ldots \ldots$ & 025607.9 & 200325 & Yes & CTTS \\
\hline $\mathrm{LkH} \alpha 263 \ldots \ldots \ldots \ldots \ldots$ & 025608.4 & 200339 & Yes & CTTS \\
\hline $\mathrm{LkH} \alpha 264 \ldots \ldots \ldots \ldots \ldots \ldots$ & 025637.5 & 200538 & Yes & CTTS \\
\hline E02553+2018 ….......... & 025811.2 & 203004 & Yes & C/WTTS \\
\hline RXJ $0258.3+1947 \ldots \ldots$. & 025815.9 & 194717 & Yes & CTTS \\
\hline RXJ 0306.5+1921 ....... & 030633.1 & 192152 & No & WTTS $^{d}$ \\
\hline S18 & 030221.1 & 171035 & Yes & CTTS $^{e}$ \\
\hline
\end{tabular}

Note.-Units of right ascension are hours, minutes, and seconds, and units of declination are degrees, arcminutes, and arcseconds.

a From Jayawardhana et al. 2001.

${ }^{\mathrm{b}}$ Defining classical T Tauri stars (CTTS) as objects having $\mathrm{H} \alpha$ equivalent line widths greater than 5-10 , and weak-line T Tauri stars (WTTS) as EW(H $\alpha)<5-10 \AA$.

c E02553 +2018 has an uncertain classification as classical or weak-line T Tauri star: it has detected infrared excess and $\mathrm{EW}(\mathrm{H} \alpha) \approx 4 \AA$.

${ }^{\mathrm{d}}$ Luhman 2001 concludes that RXJ $0306.5+1921$ is likely not a member of MBM 12 , but an older interloper.

${ }^{\mathrm{e}}$ In spite of its distance from the core of the MBM 12 cloud, Luhman 2001 conclude that $\mathrm{S} 18$ is a bona fide member.

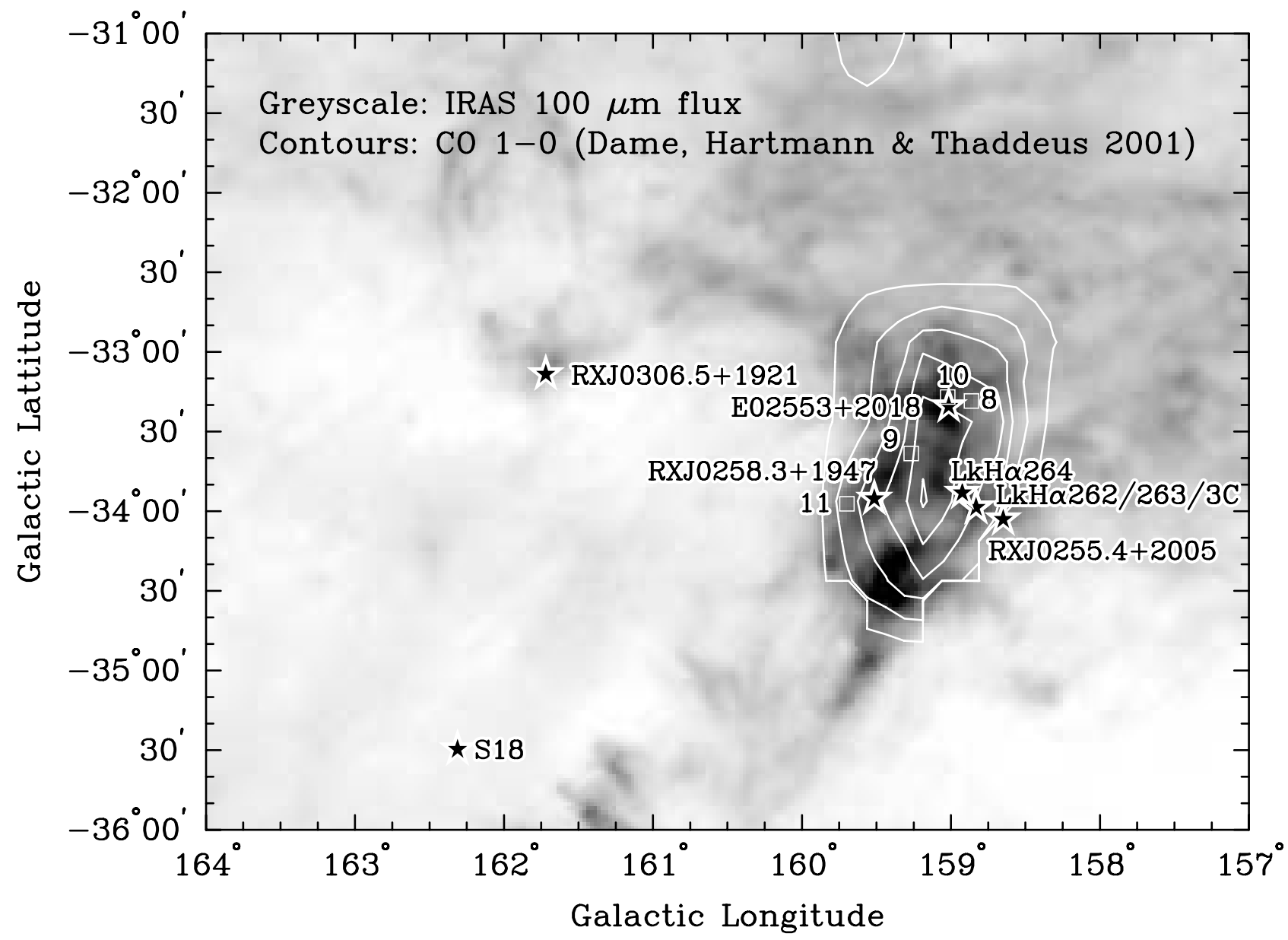

FIG. 1.-Positions of the eight MBM 12 T Tauri stars studied in this paper (stars) superposed on the IRAS $100 \mu \mathrm{m}$ emission (gray scale) and CO $J=2-1$ emission (contours, at 2, 4, 6, . K km s'-1 Dame, Hartmann, \& Thaddeus 2001). The additional four members of MBM 12 identified by Luhman 2001 are indicated by open squares. RXJ $0306.5+1921$ and S18 both are located $2^{\circ}-3^{\circ}$ from the main core of MBM 12; Luhman (2001) concludes that S18 is a true member of the association, while RXJ $0306.5+1921$ is likely an older interloper. 
along the MBM 12 line of sight, so the distance of the stars is unclear. We follow Luhman (2001) and adopt 275 pc, which gives the most plausible location of the stars on the Herzsprung-Russel diagram. In any case, the young age indicated by MBM 12's association with cloud material and its relative proximity make it an excellent target to study circumstellar disks in detail.

This paper presents the results of sensitive millimeterwave searches for cold dust and molecular gas associated with the six classical T Tauri stars in MBM 12 known to harbor disks for which no millimeter data exist, as well as the two weak-line $\mathrm{T}$ Tauri stars (Table 1). We did not include the newly identified members of MBM 12 (Luhman 2001) but fortuitously include the edge-on disk source MBM 12 3C (Jayawardhana et al. 2002), which happened to fall in our field containing $\mathrm{LkH} \alpha 262$ and $\mathrm{LkH} \alpha 263$. Since the completion of these observations, Luhman (2001) has concluded that one object, RXJ 0306.5+1921, is likely an older interloper. For completeness, we still report the data on this object. We used a millimeter interferometer to measure the continuum emission because the high spatial resolution avoids confusion with the surrounding cloud. We use single-dish submillimeter observations of ${ }^{13} \mathrm{CO} J=2-1$ to trace any cold $(\sim 16 \mathrm{~K})$ and relatively low density $\left(n_{\mathrm{H}_{2}} \approx 10^{4} \mathrm{~cm}^{-3}\right)$ gas that may reside around the objects. Section 2 describes the observations, and $\S 3$ the resulting mass limits. Section 4 discusses these limits in the context of other nearby star-forming regions and young associations. Section 5 concludes the paper with a short summary.

\section{OBSERVATIONS}

\subsection{Owens Valley Radio Observatory}

The $3 \mathrm{~mm}$ continuum observations were carried out with the Millimeter Array at the Owens Valley Radio Observatory $(\text { OVRO })^{7}$ on 2001 March 19, 20, and 24. The receivers were tuned to the frequencies of $\mathrm{C}^{34} \mathrm{~S} J=2-1$ at 96.41294 $\mathrm{GHz}$ and the $\mathrm{N}_{2} \mathrm{H}^{+} 1-0$ triplet at $93.17370 \mathrm{GHz}(\lambda=3$ $\mathrm{mm}$ ), where their performance is optimum. The lines were recorded in two $7.75 \mathrm{MHz}$-wide bands of 62 channels, while the continuum signal in the upper and lower sidebands was recorded over a total bandwidth of $2 \mathrm{GHz}$. The array was in the "E" configuration, with baselines between 10 and $40 \mathrm{k} \lambda$ resulting in a synthesized beam of $5^{\prime \prime}$. In each $8 \mathrm{hr}$ track, two sources were observed by alternating between them every half-hour. Source coordinates are listed in Table 1. $\mathrm{LkH} \alpha$ 262 and 263 are located $17^{\prime \prime}$ from each other, well within the 64 " primary beam of the $10 \mathrm{~m}$ antennas; they were observed in a single pointing for an entire $8 \mathrm{hr}$ track. The edge-on disk source MBM 12A 3C (Jayawardhana et al. 2002) is located $\sim 4^{\prime \prime}$ from $\mathrm{LkH} \alpha 263$ and also falls within the primary beam. The complex gain was calibrated by observations every 30 minutes of the nearby $\left(9^{\circ}\right)$ quasar $0235+164$ (flux $1.0 \mathrm{Jy}$ ). The phase stability was less than optimal, and only data with coherences above $\sim 60 \%$ were used.

Flux scaling was obtained from observations of Uranus and the quasar 3C 84 (flux 6.2 Jy, bootstrapped from the Uranus data). The data were calibrated in the MMA software package (Scoville et al. 1993) and further processed

\footnotetext{
${ }^{7}$ The Owens Valley Millimeter Array is operated by the California Institute of Technology with funding from the National Science Foundation (AST 96-13717).
}

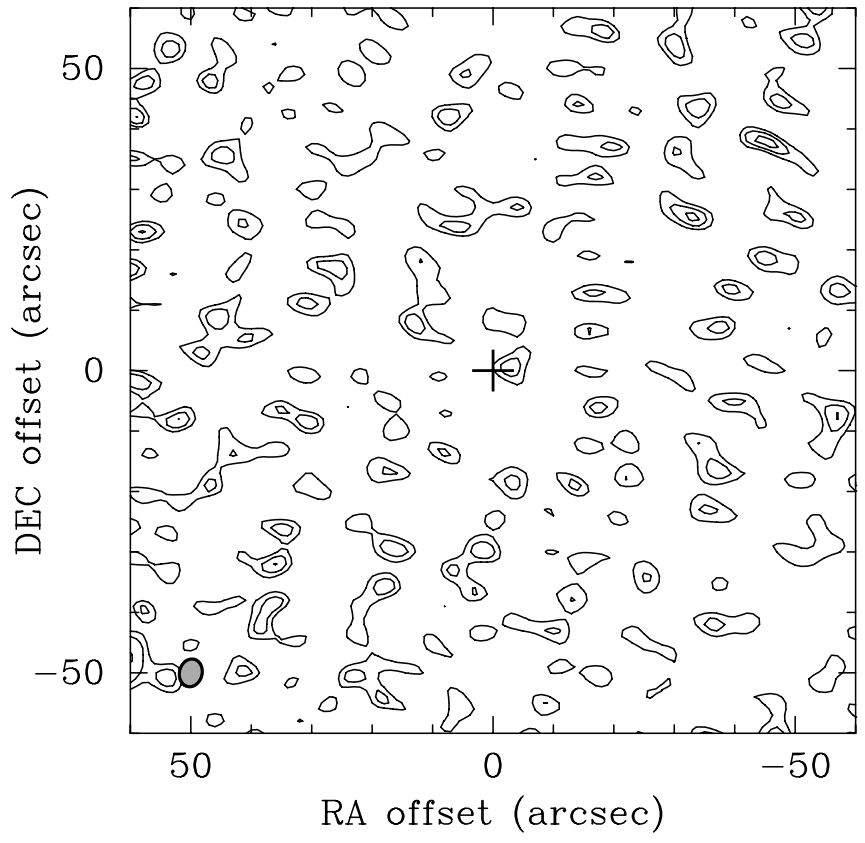

FIG. 2.-Reconstructed image of the co-added $3 \mathrm{~mm}$ continuum data from OVRO. The five observed fields have been shifted to a common origin (indicated by the cross), taking into account the source offsets for the field that contains both $\mathrm{LkH} \alpha 262$ and 263. The co-added data place an upper limit on the "average" disk mass of $0.03 M_{\odot}$ (gas plus dust). Contours are drawn at $1 \sigma=1 \mathrm{mJy}$. The synthesized beam size is 4".6 $\times 3$ ".7 and is shown in the bottom left corner. This image includes the data on the likely interloper RXJ 0306.5+1921; without these data, the resulting image is not significantly different.

with the MIRIAD package (Sault, Teuben, \& Wright 1995). The resulting $1 \sigma \mathrm{rms}$ noise level of the sideband averaged continuum was $1.3-2.9 \mathrm{mJy}$ in the $\sim 5^{\prime \prime}$ beam. We also coadded the observed five fields, aligning the source positions (Fig. 2), to derive constraints on the "average" disk mass of the six objects (see Carpenter 2002).

\subsection{James Clerk Maxwell Telescope}

The ${ }^{13} \mathrm{CO} J=2-1$ observations were carried out at the James Clerk Maxwell Telescope (JCMT) ${ }^{8}$ in Canadian service mode on 2001 March 23-25, April 9, May 24, and May 25 . The facility receiver "RxA3" was tuned to the line frequency at $220.3986765 \mathrm{GHz}$, and the spectra were recorded in Digital Autocorrelation Spectrometer back end in a bandwidth of $125 \mathrm{MHz}$ and a resolution of $78 \mathrm{kHz}(0.11 \mathrm{~km}$ $\mathrm{s}^{-1}$ ). Typical system temperatures of 400-600 K were found, giving a noise rms of $67-100 \mathrm{mK}$ per channel after onsource integration of 20 minutes; a main beam efficiency of 0.6 has been applied. The observations were carried out in a frequency switched mode with a throw of $8.2 \mathrm{MHz}$ to compensate for atmospheric contributions. The data were analyzed with the SPECX software package.

The observations were pointed at the source positions of Table 1; S18 was not observed due to time constraints. If emission was detected at that position, an integration at an offset position of $\left(+30^{\prime \prime}, 0^{\prime \prime}\right)$ was taken. If no emission at $\left(0^{\prime \prime}\right.$, $\left.0^{\prime \prime}\right)$ was detected, or if the emission at both positions was

${ }^{8}$ The JCMT is operated by the Joint Astronomy Centre, Hilo, Hawaii, on behalf of the Particle Physics and Astronomy Research Council in the United Kingdom, the National Research Council of Canada, and the Netherlands Organization for Scientific Research. 
equal within the error, we concluded that there was no molecular gas associated with the source; in the latter case, we attributed all emission to the surrounding cloud.

\section{RESULTS}

\subsection{Continuum Emission from OVRO}

The OVRO observations did not detect $3 \mathrm{~mm}$ continuum emission to $3 \sigma$ upper limits of 4-9 $\mathrm{mJy}$; for $\mathrm{LkH} \alpha 264$ and E02533+2018, Pound (1996) reported upper limits at 2.7 $\mathrm{mm}$ with a $3 \sigma$ level of 15 and $11 \mathrm{mJy}$, respectively (Table 2). No $\mathrm{C}^{34} \mathrm{~S}$ or $\mathrm{N}_{2} \mathrm{H}^{+}$emission was detected in the OVRO data at a $3 \sigma$ noise level of $\sim 0.5 \mathrm{Jy}_{\text {beam }}^{-1}(3 \mathrm{~K})$ in $0.40 \mathrm{~km} \mathrm{~s}^{-1}$ channels. These upper limits on the line emission do not provide useful constraints, and we will not analyze them further.

We derive upper limits on the mass traced by the continuum emission using the simplifying assumptions that the emission is optically thin and characterized by a single dust temperature and emission coefficient (see, e.g., Beckwith et al. 1990; Duvert et al. 2000). In the Rayleigh-Jeans limit valid for the observing wavelength and typical dust temperatures, the disk mass is related to the continuum flux as

$$
\begin{aligned}
M_{\text {disk }}= & 0.01 M_{\odot}\left(\frac{F_{\nu}(3 \mathrm{~mm})}{1 \mathrm{mJy}}\right)\left(\frac{D}{275 \mathrm{pc}}\right)^{2} \\
& \times\left(\frac{\kappa}{0.6 \mathrm{~cm}^{2} \mathrm{~g}_{\text {dust }}^{-1}}\right)^{-1}\left(\frac{T_{\text {dust }}}{25 \mathrm{~K}}\right)^{-1} .
\end{aligned}
$$

Here, $M_{\text {disk }}$ is the disk mass, $F_{\nu}(3 \mathrm{~mm})$ is the flux, $D$ is the distance, $\kappa$ is the emissivity per unit dust mass, and $T_{\text {dust }}$ is the dust temperature. $M_{\text {disk }}$ refers to the mass of the disk in gas and dust. We have adopted a gas-to-dust mass ratio of $100: 1$, as is found in the translucent interstellar medium. Since we measure the dust content only, our actual limits are $1 \times 10^{-4} M_{\odot}$ of dust per $1 \mathrm{mJy}$ of $3 \mathrm{~mm}$ flux. The adopted emissivity is a value "typical" for evolved dark clouds or protostellar disks (Beckwith \& Sargent 1991; Pollack et al. 1994; Ossenkopf \& Henning 1994) and is uncertain by a factor of a few. The dust temperature is uncertain by less than a factor of 2 . We have used the dis- tance of $275 \mathrm{pc}$; at 65 or $140 \mathrm{pc}$, the derived mass would be smaller by factors of 18 and 4 , respectively. Table 2 lists the resulting mass limits of $0.04-0.09 M_{\odot}$ (gas plus dust). This includes the values of Pound (1996) for E02553+2018 (0.07) and $\mathrm{LkH} \alpha 264\left(0.09 M_{\odot}\right)$, recalculated for our adopted dust properties and temperatures and the larger distance of 275 pc.

We can place a generous upper limit to the disk's diameter by assuming it is fully optically thick at $3 \mathrm{~mm}$ and geometrically thin. At $3 \mathrm{~mm}$, we find an upper limit of $26 \mathrm{AU}$ for a flux limit of $1 \mathrm{mJy}$, and at $2.7 \mathrm{~mm}$ of $20 \mathrm{AU}$. The resulting limits for our sample of 50-80 AU are significantly larger than the areas of circumstellar disks that are likely to be optically thick; with $\kappa=0.6 \mathrm{~cm}^{2} \mathrm{~g}^{-1}$ (dust), a 50 AU diameter fully optically thick $(\tau>3)$ disk has a mass greater than $0.1 M_{\odot}$ (gas plus dust).

We also calculated the continuum flux of a disk using more realistic descriptions by Chiang \& Goldreich (1997) and D'Alessio et al. (1998). Using the former model, scaled directly to $0.01 M_{\odot}$ without taking into account any changes to the disk's internal structure, we find a flux close to that of equation (1).

The co-added five fields yield a limit on the "average" disk mass for the six objects. We find a $1 \sigma$ rms noise level of $1 \mathrm{mJy}$, and an average disk mass below $\bar{M}_{\text {disk }}(N=5)$ $<0.03 M_{\odot}$. This average includes the data on the object RXJ 0306.5+1921, which Luhman (2001) concludes may not be a member of MBM 12. If we exclude this object, the resulting noise rms $(1.1 \mathrm{mJy})$ and $\bar{M}_{\text {disk }}$ limits $\left(0.033 M_{\odot}\right)$ do not change significantly.

\subsection{Line Emission from JCMT}

Four of our objects do not show ${ }^{13} \mathrm{CO} 2-1$ emission with a $3 \sigma$ value of $0.25 \mathrm{~K}$; the remaining have detected emission in the range of $0.7-1.3 \mathrm{~K}$ but show similar values at a position $30^{\prime \prime}$ away from the star (Table 3 and Fig. 3). Only for E02553+2018 does an additional component at $V_{\mathrm{LSR}}=-1.5 \mathrm{~km} \mathrm{~s}^{-1}$ show up at $\left(+30^{\prime \prime}, 0^{\prime \prime}\right)$, likely associated with the MBM 12 cloud. We conclude that for the latter three objects all emission comes from

TABLE 2

\begin{tabular}{|c|c|c|c|c|}
\hline Source & $\begin{array}{c}3 \sigma \text { Upper Limit } \\
\text { (mJy) }\end{array}$ & $\begin{array}{l}\text { Beam Size } \\
\text { (arcsec) }\end{array}$ & $\begin{array}{l}\text { Upper Limit } \\
\text { Disk Mass }^{\mathrm{a}} \\
\quad\left(M_{\odot}\right)\end{array}$ & $\begin{array}{l}\text { Upper Limit } \\
\text { Diameter } \\
\text { (AU) }\end{array}$ \\
\hline RXJ $0255.4+2005 \ldots \ldots .$. & 4 & $5.2 \times 4.0$ & 0.04 & 52 \\
\hline 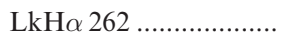 & 7 & $5.3 \times 3.5$ & 0.07 & 69 \\
\hline 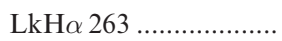 & 7 & $5.3 \times 3.5$ & 0.07 & 69 \\
\hline $\mathrm{LkH} \alpha 264 \ldots \ldots \ldots \ldots \ldots \ldots$ & $15^{\mathrm{c}}$ & $3.5 \times 3.1$ & 0.09 & 77 \\
\hline E02553+2018 ……........ & $11^{\mathrm{c}}$ & $2.7 \times 1.8$ & 0.07 & 66 \\
\hline RXJ $0258.3+1947 \ldots \ldots .$. & 5 & $5.2 \times 4.0$ & 0.05 & 58 \\
\hline RXJ 0306.5+1921 ....... & 7 & $4.9 \times 3.9$ & 0.07 & 69 \\
\hline S18 ...................... & 9 & $5.3 \times 3.9$ & 0.09 & 78 \\
\hline Average $^{d}$ & 3 & $4.6 \times 3.7$ & 0.03 & 45 \\
\hline
\end{tabular}

UPPER Limits on $\lambda=3 \mathrm{~mm}$ CONTINUUM FLUX

a Assuming a distance of $d=275 \mathrm{pc}$ and a gas-to-dust ratio of 100. Disk mass scales as $M_{d} \propto d^{-2}$ and is independent of inclination in these optically thin cases.

${ }^{b}$ Minimum diameter of a fully optically thick disk $(\tau>3)$ with a dust temperature of $25 \mathrm{~K}$ that would produce a flux comparable to the obtained upper limit.

${ }^{c}$ The $3 \sigma$ flux limits at $2.7 \mathrm{~mm}$ from Pound 1996.

d Noise level and derived upper limit on the "average" disk mass obtained by co-adding the five fields observed with OVRO, i.e., excluding LkH $\alpha 264$ and E02553+2018. 
TABLE 3

ObSERVED ${ }^{13} \mathrm{CO} J=2-1$ Line Intensities

\begin{tabular}{|c|c|c|c|}
\hline Source & $\begin{array}{c}T_{\mathrm{mb}} \\
\left(0^{\prime \prime}, 0^{\prime \prime}\right) \\
(\mathrm{K})\end{array}$ & $\begin{array}{c}T_{\mathrm{mb}} \\
\left(30^{\prime \prime}, 0^{\prime \prime}\right) \\
(\mathrm{K})\end{array}$ & $\begin{array}{l}\text { Upper Limit Mass }{ }^{\mathrm{a}, \mathrm{b}} \\
\qquad\left(10^{-4} M_{\odot}\right)\end{array}$ \\
\hline RXJ 0255.4+2005 ....... & $<0.23$ & $\ldots$ & 6 \\
\hline $\operatorname{LkH} \alpha 262$ & $<0.28$ & $\ldots$ & 7 \\
\hline $\operatorname{LkH} \alpha 263$ & $<0.40$ & $\ldots$ & 10 \\
\hline $\mathrm{LkH} \alpha 264 \ldots \ldots \ldots \ldots \ldots \ldots$ & $1.27 \pm 0.13$ & $1.03 \pm 0.1$ & 10 \\
\hline E02553+2018 _............... & $0.92 \pm 0.10$ & $1.08 \pm 0.12$ & 9 \\
\hline RXJ 0258.3+1947 ....... & $0.72 \pm 0.10$ & $0.62 \pm 0.08$ & 8 \\
\hline RXJ 0306.5+1921 ....... & $<0.22$ & $\ldots$ & 5 \\
\hline 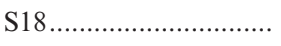 & $\ldots$ & $\ldots$ & $\cdots$ \\
\hline
\end{tabular}

a Assuming a distance of $d=275 \mathrm{pc}$. Disk mass scales as $M_{d} \propto d^{-2}$ and is independent of inclination.

${ }^{b}$ Depletion of CO by freezing out onto dust grains and/or photodissociation by (inter-) stellar ultraviolet photons can easily raise these limits by 2 orders of magnitude.

the surrounding cloud, and that the $3 \sigma$ noise level of $0.25 \mathrm{~K}$ can be adopted as upper limit to any gas mass associated with these objects.

Following Scoville et al. (1986), who relate the integrated intensity to the beam-averaged column density of a linear rotor such as ${ }^{13} \mathrm{CO}$ and using a dipole moment of $0.112 \mathrm{D}$ for ${ }^{13} \mathrm{CO}$ and $\mathrm{a}{ }^{13} \mathrm{CO} / \mathrm{H}_{2}$ abundance of $3 \times 10^{-6}$ (Lacy et al. 1994; Wilson \& Rood 1994), we

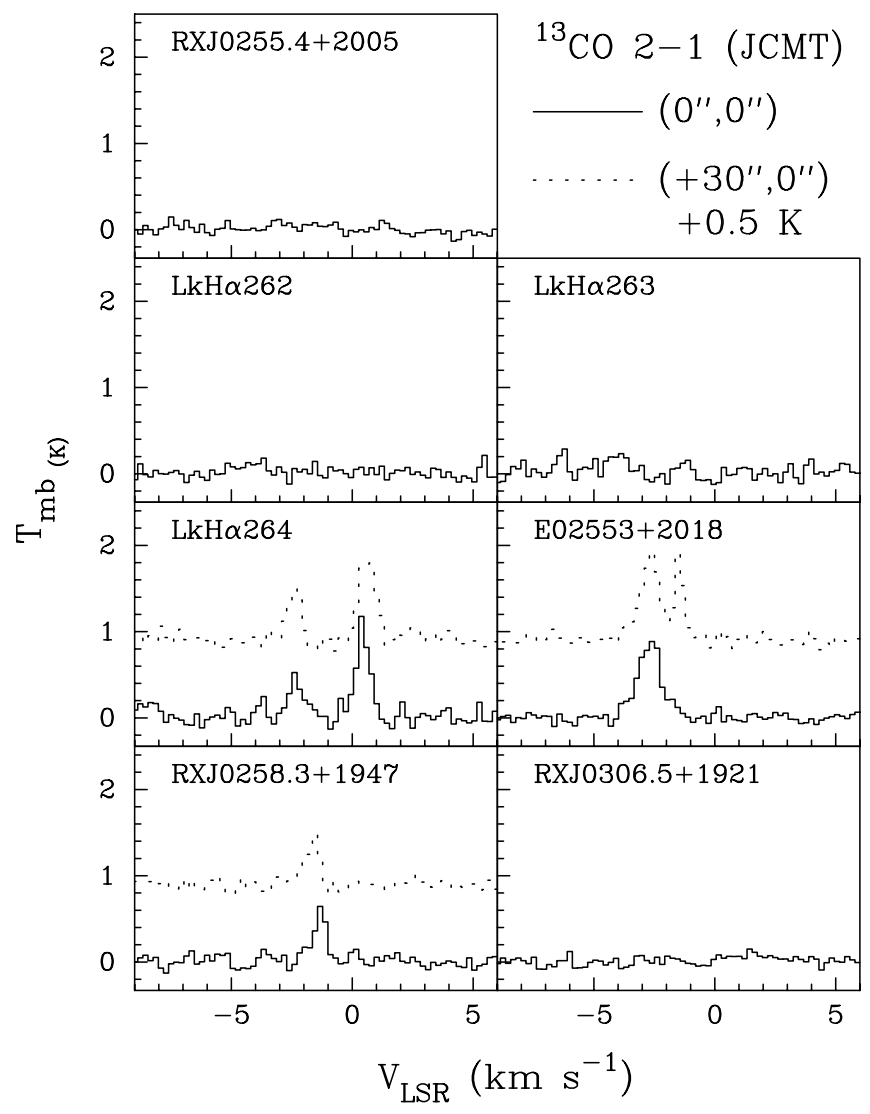

FIG. 3.-Spectra of ${ }^{13} \mathrm{CO} J=2-1$ obtained with the JCMT toward seven of our eight objects. Solid lines show the spectra to the source position. In three cases, emission was detected, and spectra at a nearby $\left(30^{\prime \prime}\right)$ cloud position were obtained (dotted lines). In all three cases, the emission was found to be equal, within the noise level, at both positions. find a disk (gas) mass of

$$
\begin{aligned}
M_{\text {disk }}= & (5-8) \times 10^{-4} M_{\odot}\left(\frac{\left[{ }^{12} \mathrm{CO}\right]:\left[\mathrm{H}_{2}\right]}{2 \times 10^{-4}: 1}\right) \\
& \times\left(\frac{\left[{ }^{12} \mathrm{CO}\right]:\left[{ }^{13} \mathrm{CO}\right]}{65: 1}\right)\left(\frac{D}{275 \mathrm{pc}}\right)^{2} \\
& \times\left(\frac{\int T_{\mathrm{mb}} d V}{0.27 \mathrm{~K} \mathrm{~km} \mathrm{~s}^{-1}}\right) .
\end{aligned}
$$

Equation (2) assumes that the emission is optically thin and uses the observed upper limit of $0.3 \mathrm{~K}$ per $0.11 \mathrm{~km}$ $\mathrm{s}^{-1}$ channel or $0.27 \mathrm{~K} \mathrm{~km} \mathrm{~s}^{-1}$ for an adopted line width of $2 \mathrm{~km} \mathrm{~s}^{-1}$. This line width is appropriate for disks in Keplerian rotation around a $0.5 M_{\odot}$ star under most inclinations. The range in masses in equation (2) reflects the considered range of excitation temperatures, $6 \mathrm{~K}<$ $T_{\mathrm{ex}}<70 \mathrm{~K}$. The relation reaches a minimum at $T_{\mathrm{ex}}=16$ $\mathrm{K}$, which corresponds to the energy of the $J=2$ level. Table 3 lists the resulting mass limits for our sample of $(5-10) \times 10^{-4} M_{\odot}$.

Using the Chiang \& Goldreich (1997) model, chemical calculations from Aikawa \& Herbst (1999), and adopting varying disk inclinations, we confirm again that a more realistic treatment of the density and temperature structure gives results consistent with equation (2).

\section{DISCUSSION}

Section 3 places upper limits on the mass of cold dust and gas associated with eight $T$ Tauri stars in MBM 12. The $3 \mathrm{~mm}$ continuum limits the mass to less than $0.04-0.09 \mathrm{M}_{\odot}$. The ${ }^{13} \mathrm{CO}$ lines provide limits that are much lower, (510) $\times 10^{-4} M_{\odot}$. However, the abundance of molecules such as $\mathrm{CO}$ may be decreased, as commonly observed in T Tauri disks (e.g., Dutrey, Guilloteau, \& Simon 1994; Dutrey et al. 1996), possibly by freezing out onto dust grains and by photodissociation by (inter-) stellar ultraviolet photons, as suggested by chemical models (e.g., Aikawa \& Herbst 1999; Willacy \& Langer 2000). These processes can easily reduce the $\mathrm{CO}$ abundance by factors of tens or a hundred, in which case the limits of Table 3 are no longer lower than those of Table 2 . We stress that if $\mathrm{CO}$ is significantly frozen out, the gas mass of the disk does not change appreciably, since it is dominated by undepleted $\mathrm{H}_{2}$. If $\mathrm{CO}$ is significantly photodissociated, the total molecular gas mass may be reduced if a sizable fraction of $\mathrm{H}_{2}$ is also photodissociated.

Jayawardhana et al. (2001) report $N$-band excess in the six classical T Tauri stars, indicating the presence of material close to the stars. This places a lower limit to the disk mass of $\sim 10^{-5} M_{\odot}$, well below our upper limits. The edgeon disk source MBM 12A 3C near $\mathrm{LkH} \alpha 263$ (Jayawardhana et al. 2002) requires a mass of $\sim 2 \times 10^{-3} M_{\odot}$ to explain its scattered light image. This mass is comfortably bracketed by our lower and upper limits. Jayawardhana et al. model the scattered light with a distribution of dust sizes. At the observing wavelength of $3 \mathrm{~mm}$, our observations are sensitive to particles much larger than those doing the infrared scattering, but both populations are connected through the adopted dust size distribution and mass emissivity coefficients and therefore refer to a similar mass reservoir.

With the obtained limits, the disk masses in MBM 12 are indistinguishable from those found in Taurus-Auriga and $\rho$ Oph of $0.001-0.3 M_{\odot}$ (Beckwith et al. 1990; Osterloh \& 
Beckwith 1995; André \& Montmerle 1994). We therefore cannot draw any conclusions about the evolution of disks in the 1-2 Myr age range spanned by these regions and MBM 12. While the work by Luhman (2001) suggests that the dust in the disks in MBM 12 is starting to clear out, more sensitive measurements of the dust continuum with, e.g., SCUBA or SIRTF are needed to investigate the fate of the colder dust at larger radii. Carpenter (2002) limits the disk masses of the members of the $2 \mathrm{Myr}$ cluster IC 348 to less than $0.025 M_{\odot}$ (or $0.002 M_{\odot}$ averaged over his 95 sources), somewhat below our limits on MBM 12.

Our mass limits do exclude the presence of objects such as T Tau, GG Tau, and DG Tau with large millimeter fluxes (Beckwith et al. 1990). We would easily have detected these fluxes at 20-40 mJy when scaled to $275 \mathrm{pc}$ and extrapolated to $3 \mathrm{~mm}$ (adopting spectral indexes between 2.5 and 4). However, even in Taurus-Auriga these objects are rare and given the small number of T Tauri stars in MBM $12(\sim 10$; Luhman 2001), no such bright objects would necessarily be expected.

\section{SUMMARY}

We obtained upper limits on the $3 \mathrm{~mm}$ continuum flux and ${ }^{13} \mathrm{CO} 2-1$ line intensity of eight $\mathrm{T}$ Tauri stars in the
MBM 12 region. These limits constrain the disk masses to less than 0.04-0.09 $M_{\odot}$ (gas plus dust), consistent with the distribution of masses in slightly younger regions like Taurus-Auriga and $\rho$ Oph ( $\sim 1$ Myr vs. $\sim 2 \mathrm{Myr})$, and consistent with the mass of $2 \times 10^{-3} M_{\odot}$ derived for the edge-on disk of MBM 12A 3C. We exclude the presence of objects such as T Tau, GG Tau, and DG Tau with bright millimeter emission. More sensitive searches with, e.g., SCUBA and SIRTF will probe the evolution of the cold disk material at larger radii at the moment when the inner disks start to clear.

We wish to thank the staff of OVRO and JCMT, and Henry Matthews in particular, for their outstanding support. Paola D'Alessio, Erik Mamajek, Michael Meyer, and James Muzerolle are thanked for useful discussions. The referee provided many useful comments that significantly improved the paper. At the University of California, Berkeley, the research of M. R. H. and R. J. was supported by the Miller Institute for Basic Research in Science. This work was supported in part by NASA Origins grant NAG 511905 to R. J.
Aikawa, Y., \& Herbst, E. 1999, A\&A, 351, 233

André, P., \& Montmerle, T. 1994, ApJ, 420, 837

Beckwith, S. V. W., \& Sargent, A. I. 1991, ApJ, 381, 250

Beckwith, S. V. W., Sargent, A. I., Chini, R. S., \& Güsten, R. 1990, AJ, 99 924

Carpenter, J. M. 2002, AJ, 124, 1593

Chiang, E. I., \& Goldreich, P. 1997, ApJ, 490, 368

D’Alessio, P., Canto, J., Calvet, N., \& Lizano, S. 1998, ApJ, 500, 411

Dame, T. M., Hartmann, D., \& Thaddeus, P. 2001, ApJ, 547, 792

Dutrey, A., Guilloteau, S., Duvert, G., Prato, L., Simon, M., Schuster, K., \& Ménard, F. 1996, A\&A, 309, 493

Dutrey, A., Guilloteau, S., \& Guélin, M. 1997, A\&A, 317, L55

Dutrey, A., Guilloteau, S., Prato, L., Simon, M., Duvert, G., Schuster, K., \& Ménard, F. 1998, A\&A, 338, L63

Dutrey, A., Guilloteau, S., \& Simon, M. 1994, A\&A, 286, 149

Duvert, G., Guilloteau, S., Ménard, F., Simon, M., \& Dutrey, A. 2000 A\&A, 355, 165

Haisch, K. E., Lada, E. A., \& Lada, C. J. 2001, ApJ, 553, L153

Hartmann, L. 2001, AJ, 121, 1030

Hearty, T., Fernández, M., Alcalá, J. M., Covino, E., \& Neuhäuser, R. 2000a, A\&A, 357, 681

Hearty, T., Neuhäuser, R., Stelzer, B., Fernández, M., Alcalá, J. M., Covino, E., \& Hambaryan, V. 2000b, A\&A, 353, 1044

Herczeg, G. J., Linsky, J. L., Valenti, J. A., Johns-Krull, C. M., \& Wood, B. E. 2002, ApJ, 572, 310

Idzi, R., Andersson, B.-G., \& Uomoto, A. 2001, BAAS, 33, 1449

Jayawardhana, R. 2000, Science, 288, 64

Jayawardhana, R., \& Greene, T., ed. 2001, ASP Conf. Ser. 244, Young

Stars Near Earth: Progress and Prospects (San Francisco: ASP)

Jayawardhana, R., Hartmann, L., Fazio, G., Fisher, R. S., Telesco, C. M., \& Piña, R. K. 1999, ApJ, 521, L129

Jayawardhana, R., Luhman, K. L., D’Alessio, P., \& Stauffer, J. R. 2002, ApJ, 571, L51

Jayawardhana, R., Wolk, S. J., Barrado y Navascués, D., Telesco, C. M., \&

Hearty, T. J. 2001, ApJ, 550, L197

\section{REFERENCES}

Kastner, J. H., Zuckerman, B., Weintraub, D. A., \& Forveille, T. 1997, Science, 277, 67

Lacy, J. H., Knacke, R., Geballe, T. R., \& Tokunaga, A. T. 1994, ApJ, 428, L69

Luhman, K. L. 2001, ApJ, 560, 287

Mamajek, E. E., Lawson, W. A., \& Feigelson, E. D. 1999, ApJ, 516, L77

Mannings, V., \& Sargent, A. I. 1997, ApJ, 490, 792 2000, ApJ, 529, 391

Ossenkopf, V., \& Henning, T. 1994, A\&A, 291, 943

Osterloh, M., \& Beckwith, S. V. W. 1995, ApJ, 439, 288

Pollack, J. B., Hollenbach, D., Beckwith, S. V. W., Simonelli, D. P., Roush, T., \& Fong, W. 1994, ApJ, 421, 615

Pound, M. W. 1996, ApJ, 457, L35

Richter, M. J., Jaffe, D. T., Blake, G. A., \& Lacy, J. H. 2002, ApJ, 572, L161

Robberto, M., Beckwith, S. V. W., \& Herbst, T. M. 2000, in Star Formation 1999, ed. T. Nakamoto (Nobeyama: Nobeyama Radio Obs.), 231

Sandell, G., Reipurth, B., \& Gahm, G. 1987, A\&A, 181, 283

Sault, R. J., Teuben, P. J., \& Wright, M. C. H. 1995, in ASP Conf. Ser. 77, Astronomical Data Analysis Software and Systems IV, ed. R. A. Shaw, H. E. Payne, \& J. J. E. Hayes (San Francisco: ASP), 433

Scoville, N. Z., Carlstrom, J. E., Chandler, C. J., Phillips, J. A., Scott, S. L., Tilanus, R. P. J., \& Wang, Z. 1993, PASP, 105, 1482

Scoville, N. Z., Sargent, A. I., Sanders, D. B., Claussen, M. J., Masson, C. R., Lo, K. Y., \& Phillips, T. G. 1986, ApJ, 303, 416

Simon, M., Dutrey, A., \& Guilloteau, S. 2000, ApJ, 545, 1034

Thi, W.-F., et al. 2001a, Nature, 409, 60

Thi, W.-F., van Dishoeck, E. F., Blake, G. A., van Zadelhoff, G., \& Hogerheijde, M. R. 1999, ApJ, 521, L63

Thi, W. F., et al. 2001b, ApJ, 561, 1074

Willacy, K., \& Langer, W. D. 2000, ApJ, 544, 903

Wilson, T. L., \& Rood, R. 1994, ARA\&A, 32, 191 